\title{
The effect of number of response categories on unidimensional concept identification
}

\author{
WILLIAM J. THOMSON and ALBERT L. PORTERFIELD \\ University of Michigan, Dearborn, Michigan 48128
}

\begin{abstract}
Concept identification problems with a single relevant dimension may be solved in two stages: the selection of the relevant dimension and the learning of appropriate responses to the values of the dimension. Such a theory was investigated by manipulating the number of response categories (four, six, or eight) in a 3 by 3 design involving 90 human subjects. Errors were found to increase with increases in response categories during all stages of problem solution, with a linear increase for paired associate and complete learning conditions. The results were interpreted as supporting a theory incorporating overlapping dimension selection and paired associate processes.
\end{abstract}

Several theories have been proposed that complete learning $(\mathrm{CL})$ of concept identification problems involves two stages, dimension selection (DS) and paired association learning (PA) (see Thomson, 1972, for a review). Although details differ, these models essentially propose that the subject randomly samples one (if the problem is simple) or two (if the problem is conjunctive) dimensions and associates values from these dimensions with the current response.The association process then continues until the problem is solved or until error feedback indicates that the sampled dimensions are incorrect, at which time resampling occurs.

Such models lead to several straightforward predictions regarding the effect of manipulating the number of response categories in the problem. The basic predictions are threefold. First, if DS is a random selection process that occurs only after errors, the number of errors in DS should be relatively independent of the number of response categories. (It may be shown that a slight positive relationship between errors in DS and number of response categories should occur, but relative to the effect of number of responses on the PA stage, the DS relationship is minimal.) In conjunctive tasks, both Overstreet and Dunham (1969) and Thomson (1972) have found DS to be independent of responses.

The second prediction involves the PA process. In this stage the essential characteristic of the models is an all-or-none learning process that predicts a linear increase in errors as a function of increasing response categories. Furthermore, if DS is independent of number of responses, and if $C L$ consists of a summation of DS and PA, then CL should also exhibit a linear increase in errors. In conjunctive tasks Thomson (1972) found the expected linearity. However, data from Polson and Dunham (1971) indicate a negatively accelerating function in a simple concept identification task.

A final prediction relates to the estimation of CL data from independent DS and PA conditions. If DS precedes and is complete before PA, then errors in CL should be a simple summed function of the errors in DS and PA. Thomson (1972), using a four-dimension conjunctive task, found a constant difference in trials to criterion between PA and CL as a function of number of response categories, implying a constant DS solution time. However, trials to criterion of an independent DS group failed to match the difference obtained by subtracting PA trials from CL trials.

The purpose of the present experiment was to test these predictions using a simple concept identification task with a single relevant dimension. Three basic groups representing DS, PA, and CL were shown identical series of stimuli and given appropriate response category feedback. The task in DS was to record on each trial which dimension was thought to be the relevant dimension, while in both PA and CL conditions, subjects were required to write down the appropriate response category. PA and CL conditions differed in that PA subjects were told the relevant dimension at the outset of the experiment. Subgroups in each condition received problems containing four, six, or eight response categories.

\section{METHOD}

\section{Subjects and Stimuli}

The subjects were 90 introductory psychology students participating in partial fulfillment of a course requirement.

Stimuli were slides of geometric forms varying along three dimensions: color (red, yellow, blue, green, brown, gray, lavender, orange), form (square, circle, triangle, cross, hexagon, star, parallelogram, diamond), and position of a black dot around the periphery of the figure (north, south, east, west, $\mathrm{NW}, \mathrm{SE}, \mathrm{SE}, \mathrm{SW}, \mathrm{NE}$ ). In conditions in which four (or six) values were used from a dimension, the first four (or six) values listed above were utilized.

\section{Procedure}

Three instruction conditions, DS, PA, and CL, were used, and each instruction condition had subgroups containing four, six, or eight response categories. Thus a total of nine experimental groups were constructed: DS 4 , DS-6, DS-8, PA-4, PA-6, PA-8, CL-4, CL-6, and CL-8.

Detailed instructions indicating that the experiment was a 
straightforward classification problem were given to all subjects. Two stimulus slides illustrating different values from each of the three dimensions were shown simultaneously to familiarize subjects with the dimensions. The names (but not the values) of each dimension were available to subjects throughout the experiment.

The subject was told that the slides had been classified by being sorted into four (six or eight) categories on the basis of values from one and only one of the dimensions. The classification rule was illustrated with hypothetical examples using each of the three dimensions and their associated values. Emphasis was placed on understanding the classification rule, and subjects were reminded that all slides were classified on the basis of values from one dimension that would remain unchanged throughout the experiment.

Subjects in Groups CL and PA were instructed to indicate the classification of each slide by responding with the name of the response category. Subjects in Group PA were also told which dimension was relevant (always color). Subjects in Group DS responded to each stimulus by recording the dimension upon which they felt the classification was based. After responding, subjects in each group were given correct response category feedback and were allowed to examine the slide for a few seconds before proceeding. Presentation was essentially self-paced, with all subjects receiving 72 trials. Three to 16 subjects were run simultaneously.

Three orders of 72 stimulus slides were constructed, one for each number of response category condition. So that inherent advantages of one condition over another would be minimized, and so that the orders would be as similar as possible, stimuli for each order were randomly generated under the following restrictions: (1) One dimension had eight values, one six, and one four; (2) consecutive stimuli had no values in common; (3) at least two stimuli intervened between the occurrence of two stimuli from the same response category; and (4) stimuli were generated in cycles, where a cycle is defined as the occurrence of a stimulus from each possible response category. Since detailed information about the above restrictions could have aided in problem solution, such information was withheld in the instructions.

\section{RESULTS}

\section{Condition DS}

Analysis of variance performed on the number of errors in selecting the relevant dimension indicated a significant response category effect $[\mathrm{F}(2,29)=8.3$, $\mathrm{p}<.01]$ (see Figure 1). Pairwise comparision revealed significantly fewer errors in the four-response category condition (5.8) than either the six-response condition (25.5) $[\mathrm{F}(1,27)=13.0, \mathrm{p}<.01]$ or the eight-response condition $(24.5)[F(1,27)=11.7, p<.01]$. No differences were found between the six- and eight-response conditions.

\section{Conditions CL and PA}

An analysis of variance for total response category errors revealed significant main effects for number of response categories $[F(2,54)=14.8, p<.001]$ and instructions $[\mathrm{F}(1,54)=37.6, \mathrm{p}<.001]$; the interaction was nonsignificant (Figure 1). The means for the PA condition for four-, six-, and eight-response categories were $3.0,9.6$, and 13.4, respectively. Corresponding means for the CL condition were 10.6, 30.9, and 35.7. A test for linearity in the PA condition indicated that

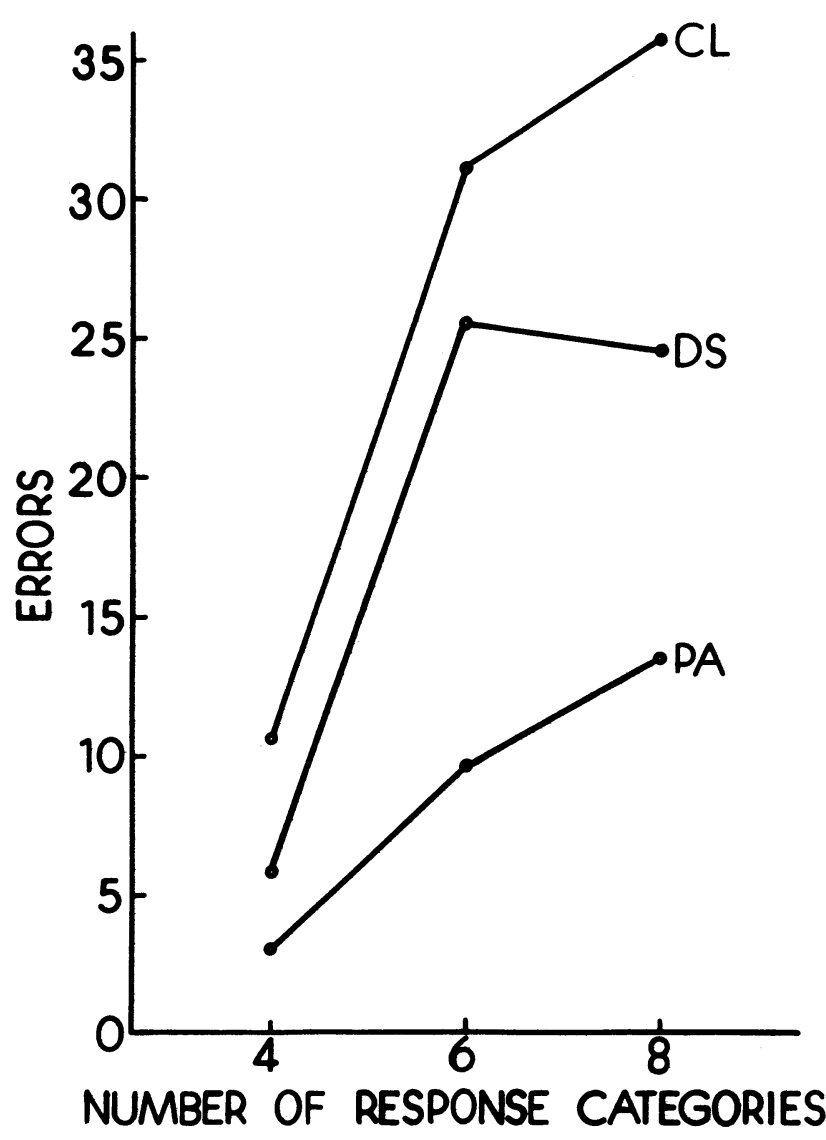

Figure 1. Total errors as a function of number of response categories.

$97.6 \%$ of the variance $[F(1,27)=36.6, p<.001]$ was accounted for by the linear component.

\section{DISCUSSION}

The prediction that DS would be unaffected by manipulating the number of response categories was not upheld. While the predicted equality was found between the six-and eight-response conditions, the four-response groups performed significantly better than either the six- or eight-response groups. The performance of the four-response groups may in part be due to the restrictions placed on sequence construction, which resulted in pairs of stimuli from the same response category having a mean number of three, five, or seven intervening stimuli for the four-, six-, and eight-response conditions, respectively. Since memory for previous information has been shown to aid problem solution (Dominowski, 1965), and since items from the same response category may be used to deduce the relevant dimension, any condition in which memory for two items from the same category exists should lead to faster solution. For only the fourresponse condition to meet this criterion, memory would need to exist for at least three or four items, and memory for more than five items should be minimal. Coltheart (1971) found that subjects could correctly recall 3.8 out of 8 stimuli in a concept identification experiment, and Atkinson and Shiffrin (1968) described a series of paired associate tasks in which working memory was found to contain two to five items simultaneously. While both the Coltheart and the Atkinson and Shiffrin studies were quite different from the present experiment, it is tenable that the current four-response condition may have differentially 
benefited by occasionally having items from the same response category simultaneously present in working memory.

Support for the prediction of linearity of errors as a function of the number of responses in the PA condition was found. In addition, significant linearity of errors was also found in the CL condition $[\mathrm{F}(1,27)=14.5, \mathrm{p}<.001]$, as would be expected if DS were independent of response categories.

The final prediction of independent and sequential DS and PA processes received limited support. While errors in DS were not found to be totally independent of response categories, the lack of an Instructions by Response Category interaction for the PA and CL conditions is consonant with the notion of a constant difference between PA and CL errors, which in turn implies constant DS errors.

However, a closer examination of the error relationships between the three instruction conditions casts considerable doubt on the notion that DS precedes and is complete before PA begins. If this assumption is true, one may assume that the relevant dimension is not known on any trial prior to or including the trial of last error (TLE) in DS. Thus, in order to translate the DS errors into response errors, all trials up to and including the TLE were multiplied by the appropriate probability of guessing an incorrect response (e.g., for the eight-response condition, TLE was multiplied by $7 / 8$ ). Further, assuming that the relevant dimension was known on trials following the TLE, the PA data were used to estimate the expected number of errors in the trials following the TLE up to a total of 72 trials (since CL was terminated at 72 trials). If DS and PA are sequential and independent, addition of these two estimates should approximate the errors in CL. Such analysis predicts 8.3, 40.6, and 43.6 response errors in CL for the four-, six-, and eight-response conditions, whereas the CL data revealed $10.6,30.9$, and 35.7 errors, respectively. Clearly, subjects made fewer errors in CL than predicted by summed DS and PA data, which implies that DS and PA may overlap rather than be sequentially independent.

A simple overlap model that assumes that the PA process occurs on all trials in which the relevant dimension was chosen, whether prior to the TLE or not, was then tested. Again, the model utilizes DS and PA data to predict CL performance. Consider the subjects in DS-8 who made nine correct dimension selections prior to the TLE and a total of 24 errors (TLE $=9+$ $24=33$ ). Estimates of the response errors were assumed to consist of two parts: response errors due to erroneous DS and response errors due to PA. First, consider response errors due to erroneous DS (e.g., 24 trials). If guessing is assumed, the expected number of response errors would be $7 / 8 \times 24=21$. An estimate of the number of response errors due to PA was obtained directly from the PA data by taking, using the present example, the number of errors in the first nine PA trials (e.g., 7.3). It should be noted that it is assumed that the PA errors occur prior to the completion of DS; that is, DS and PA overlap. Thus, for the above subject, a total of $21+7.3=28.3$ response errors would be expected through the TLE.

Following the TLE, PA is assumed to continue, and the expected number of response errors was again estimated from the PA data. Since all subjects received 72 trials, and since errors for the first 33 trials have already been estimated for the above subjects, 47 trials remain for further error estimation. Since nine trials were used to estimate response errors during DS, remaining errors were estimated from PA Trials 10-56. In these 47 trials the PA data indicated that 6.1 response errors occurred. Thus, a CL subject corresponding to the above DS subject should make $28.3+6.1=34.4$ response errors. Similar estimates were made for each subject in DS, resulting in estimated CL errors of 7.4 (observed $=10.6), 30.3$ (observed $=30.9)$, and 33.3 (observed $=$ 35.7) for Conditions 4, 6, and 8, respectively. Clearly, this simple overlap assumption approximates the data much more accurately than does an independent sequential assumption.

In general, the present study supports the duoprocess predictions. DS was relatively independent of number of responses, and PA errors were linear, as expected. However, a strict dichotomy was not supported, and support was found for overlapping DS and PA processes.

\section{REFERENCES}

Atrinson, R. C., \& Shiffrin, R. M. Human memory: A proposed system and its control processes. In K. W. Spence \& J. T. Spence (Eds.), The psychology of learning and motivation: Advances in theory and research (Vol. 2). New York: Academic Press, 1968.

Colthe ART, V. Memory for stimuli and memory for hypotheses in concept identification. Journal of Experimental Psychology, 1971, 89, 102-108.

Dominowski, R. L. Role of memory in concept learning. Psychological Bulletin, 1965, 63, 271-280.

Overstreet, J. L., \& Dunham, J. L. Effects of number of values and irrelevant dimensions on dimension selection and associative learning in a multiple concept problem. Journal of Experimental Psychology, 1969, 79, 265-268.

Polson, P. G., \& Dunham, J. L. A comparison of two types of theories of multiple-category concept identification. Journal of Verbal Learning and Verbal Behavior, 1971, 10, 618-624.

Tномson, W. J. Effect of number of response categories on dimension selection, paired-associate learning, and complete learning in a conjunctive concept identification task. Journal of Experimental Psychology, 1972, 93, 95-99.

(Received for publication December 11, 1979) 